\title{
Motion compensated whole-heart coronary cardiovascular magnetic resonance angiography using focused navigation (fNAV)
}

Christopher W. Roy ${ }^{1 *}$ (D) John Heerfordt ${ }^{1,2}$, Davide Piccini ${ }^{1,2}$, Giulia Rossi ${ }^{1}$, Anna Giulia Pavon³, Juerg Schwitter ${ }^{3,4,5}$ and Matthias Stuber ${ }^{1,6}$

\begin{abstract}
Background: Radial self-navigated (RSN) whole-heart coronary cardiovascular magnetic resonance angiography (CCMRA) is a free-breathing technique that estimates and corrects for respiratory motion. However, RSN has been limited to a 1D rigid correction which is often insufficient for patients with complex respiratory patterns. The goal of this work is therefore to improve the robustness and quality of 3D radial CCMRA by incorporating both 3D motion information and nonrigid intra-acquisition correction of the data into a framework called focused navigation (fNAV).

Methods: We applied fNAV to 500 data sets from a numerical simulation, 22 healthy subjects, and 549 cardiac patients. In each of these cohorts we compared fNAV to RSN and respiratory resolved extradimensional golden-angle radial sparse parallel (XD-GRASP) reconstructions of the same data. Reconstruction times for each method were recorded. Motion estimate accuracy was measured as the correlation between fNAV and ground truth for simulations, and fNAV and image registration for in vivo data. Percent vessel sharpness was measured in all simulated data sets and healthy subjects, and a subset of patients. Finally, subjective image quality analysis was performed by a blinded expert reviewer who chose the best image for each in vivo data set and scored on a Likert scale $0-4$ in a subset of patients by two reviewers in consensus.
\end{abstract}

Results: The reconstruction time for fNAV images was significantly higher than RSN (6.1 \pm 2.1 min vs $1.4 \pm 0.3$, min, $p<0.025)$ but significantly lower than XD-GRASP $(25.6 \pm 7.1$, min, $p<0.025)$. Overall, there is high correlation between the fNAV and reference displacement estimates across all data sets $(0.73 \pm 0.29)$. For simulated data, healthy subjects, and patients, fNAV lead to significantly sharper coronary arteries than all other reconstruction methods $(p<0.01)$. Finally, in a blinded evaluation by an expert reviewer fNAV was chosen as the best image in 444 out of 571 data sets $(78 \% ; p<0.001)$ and consensus grades of fNAV images $(2.6 \pm 0.6)$ were significantly higher $(p<0.05)$ than uncorrected $(1.7 \pm 0.7)$, RSN (1.9 \pm 0.6$)$, and XD-GRASP (1.8 \pm 0.8$)$.

Conclusion: $\mathrm{fNAV}$ is a promising technique for improving the quality of RSN free-breathing 3D whole-heart CCMRA. This novel approach to respiratory self-navigation can derive 3D nonrigid motion estimations from an acquired 1D signal yielding statistically significant improvement in image sharpness relative to $1 \mathrm{D}$ translational correction as well as XD-GRASP reconstructions. Further study of the diagnostic impact of this technique is therefore warranted to evaluate its full clinical utility.

\footnotetext{
*Correspondence: christopher.roy@chuv.ch

${ }^{1}$ Department of Radiology, Lausanne University Hospital (CHUV)

and University of Lausanne (UNIL), Rue de Bugnon 46, BH-7-84,

1011 Lausanne, Switzerland

Full list of author information is available at the end of the article
} permits use, sharing, adaptation, distribution and reproduction in any medium or format, as long as you give appropriate credit to the original author(s) and the source, provide a link to the Creative Commons licence, and indicate if changes were made. The images or other third party material in this article are included in the article's Creative Commons licence, unless indicated otherwise in a credit line to the material. If material is not included in the article's Creative Commons licence and your intended use is not permitted by statutory regulation or exceeds the permitted use, you will need to obtain permission directly from the copyright holder. To view a copy of this licence, visit http://creativecommons.org/licenses/by/4.0/. The Creative Commons Public Domain Dedication waiver (http://creativeco mmons.org/publicdomain/zero/1.0/) applies to the data made available in this article, unless otherwise stated in a credit line to the data. 
Keywords: Coronary magnetic resonance angiography, Motion correction, Whole heart, Free-breathing

\section{Background}

Whole-heart coronary cardiovascular magnetic resonance angiography (CCMRA) is a non-invasive alternative to $\mathrm{X}$-ray coronary angiography, providing high resolution assessment of complex cardiac structures without exposure to ionizing radiation $[1,2]$. In conventional CCMRA, electrocardiogram (ECG) triggering is used to limit data collection to mid-diastole, effectively "freezing" cardiac motion [3]. Additionally, a one dimensional (1D) navigator echo is typically prescribed over the dome of the liver to monitor the primary direction of respiratory motion $[4,5]$ and further limit data collection to a small acceptance window manually defined at end-expiration. Unfortunately, prospective gating is often impeded by physiological variability, resulting in inefficient and unpredictable scan times which has led to several proposed strategies for freebreathing CCMRA.

Among the alternatives to conventional prospective respiratory motion tracking and data rejection is radial self-navigated (RSN) whole-heart CCMRA [6]. In RSN CCMRA, three-dimensional (3D) radial k-space data are acquired throughout the entire respiratory cycle and a readout orientated along the superior-inferior (SI) direction is repeated at the beginning of each radial interleave. In this way, the movement of the heart due to respiration can be quantified and corrected for in a patient-specific manner by measuring the relative correlation between Fourier transformed superior-inferior (SI) readouts over a region of interest containing the blood pool. RSN CCMRA, using a spiral phyllotaxis distribution of the radial readouts $[7,8]$, has been shown to provide high-quality images with isotropic resolution in patient studies [9-11] and improved scanning efficiency relative to conventional prospectively navigated Cartesian CCMRA [12].

Still, the existing approach for RSN is limited to respiratory motion quantification in one-dimension (1D) along the SI direction which is often insufficient for patients with significant respiratory motion along the anterior-posterior (AP) or left-right (LR) directions. Furthermore, subsequent rigid correction of the $\mathrm{k}$-space data can not account for the non-linear behavior of respiratory motion. As a result, an alternative reconstruction of 3D radial CMRA data was proposed wherein a respiratory signal is derived from repeated SI readouts, the k-space data are sorted in to multiple respiratory states and reconstructed as respiratory resolved images using eXtra-Dimensional Golden-angle
RAdial Sparse Parallel (XD-GRASP) CMR [13, 14]. While it has been shown that XD-GRASP provides sharper images than the $1 \mathrm{D}$ correction scheme, this approach may be adversely affected by residual uncorrected intra-bin motion, overregularization, and long computation times [15].

The goal of this work is therefore to improve the robustness and quality of respiratory self-navigated 3D radial CCMRA by incorporating both 3D motion information and nonrigid intra-acquisition correction of the data. To do this, we propose a novel framework hereafter referred to as focused navigation (fNAV). Our approach integrates the following three features: (i) a 3D radial CCMRA acquisition with periodically repeated SI readouts, (ii) an autofocusing-based algorithm [16-20] that converts a unitless 1D respiratory signal derived from SI readouts into displacement fields along all three spatial dimensions with physical units, and (iii) an iterative reconstruction that optimizes both local image sharpness and smoothness in the displacement fields, resulting in a final $3 \mathrm{D}$ image that is regionally corrected for intraacquisition respiratory motion.

To evaluate the fNAV framework, we present a comprehensive numerical simulation that provides ground truth references for displacement field estimates and image reconstructions. We then demonstrate the use of fNAV in healthy subjects and in a large cohort of cardiac patients. Finally, we test the hypothesis that 3D nonrigid respiratory motion correction using fNAV improves coronary vessel sharpness relative to previously reported methods for 1D corrected RSN [8] and respiratory resolved XD-GRASP reconstructions of the same data sets $[13,14]$.

\section{Methods \\ Respiratory signal extraction}

The fNAV framework is applied to a previously described prototype respiratory self-navigated ECG-triggered 3D balanced stead state free precession (bSSFP) sequence with spiral phyllotaxis radial sampling, T2-preperation, spatial pre-saturation, and fat saturation pulses [7-9]. At the beginning of each radial interleave, a readout orientated along the SI direction is used for self-navigation (Fig. 1a). To extract a respiratory signal (Fig. 1b), principal component analysis is applied to a matrix of SI projections (Fourier transform of each SI readout) from each receiver coil. The strongest principal component within the expected respiratory frequency range is chosen as the respiratory signal $[14,21]$. 
a

Acquire 3D Radial Data with

Phyllotaxis Sampling
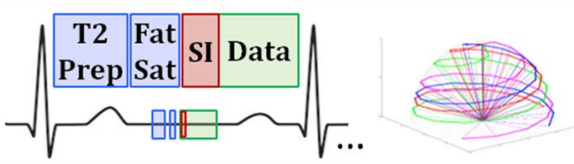

b

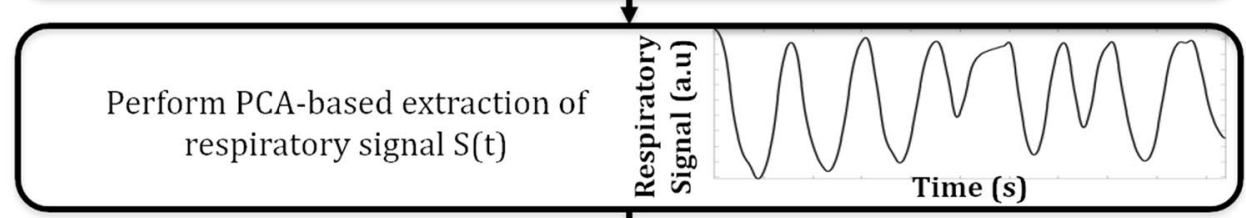

c

Calculate displacement from resp. signal and fNAV coefficients

Displacement $_{\mathrm{x}}=\mathrm{S}(\mathrm{t}) \cdot \mathbf{A}_{\mathrm{x}}$

Displacement $_{\mathrm{y}}=\mathrm{S}(\mathrm{t}) \cdot \mathrm{A}_{\mathrm{y}}$

Displacement $_{\mathrm{z}}=\mathrm{S}(\mathrm{t}) \cdot \mathrm{A}_{\mathrm{z}}$

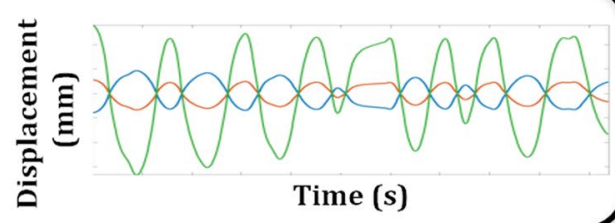

Correct k-space

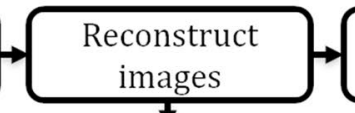

Calculate image metric

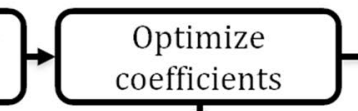

d Create a bank of fNAV coefficient $\mathbf{B}_{\mathbf{A}}$, image reconstructions $\mathbf{B}_{\mathbf{I}}$ and metric values $B_{H}$ corresponding to $m$ tested values of $A_{x} A_{y} A_{z}$

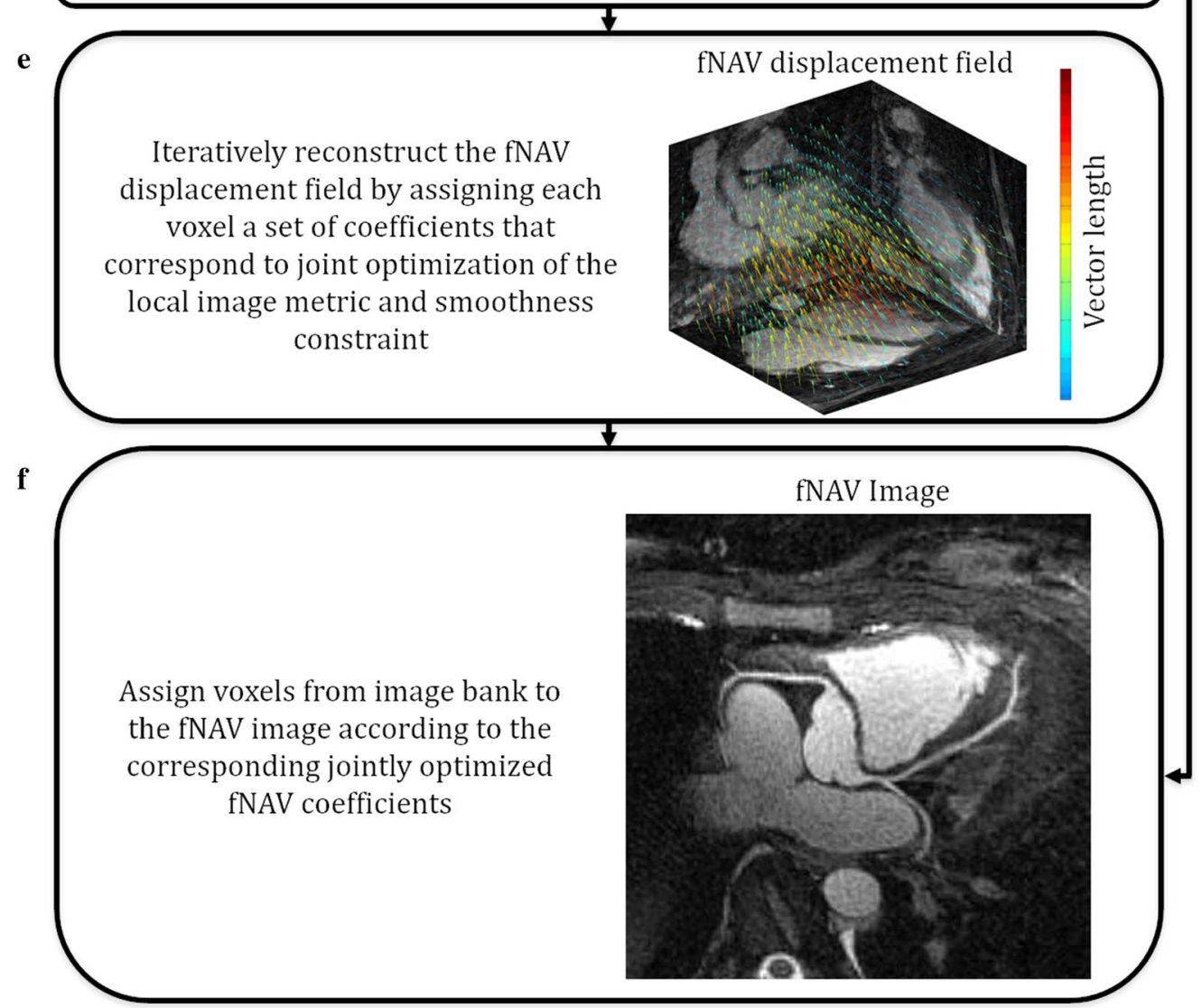

Fig. 1 Schematic overview of the focused navigation framework 


\section{Focused navigation}

It is well established that SI-derived respiratory signals $[6,8]$ are proportional to bulk motion within the sensitivity range of the receiver coils or the imaging volume for non-selective and selective excitations respectively. However, such signals are unitless and therefore require a patient-specific calibration or iterative algorithm to measure physical displacement [8, 22]. The proposed fNAV framework (Fig. 1c) posits that patient-specific 3D displacement can be approximated by a normalized SIderived respiratory signal $\mathrm{S}(\mathrm{t})$ measured at time points (t) multiplied with fNAV coefficients $\mathbf{A}(\mathbf{r})=\left[\mathrm{A}_{\mathrm{x}}(\mathrm{r}), \mathrm{A}_{\mathrm{y}}(\mathrm{r})\right.$, $A_{z}(r)$ ] that describe the millimetric amplitude of respiratory motion for a given spatial location $\mathbf{r}=[\mathrm{x}, \mathrm{y}, \mathrm{z}]$. Using, these coefficients, an image $\left(\mathrm{I}_{\mathrm{r}}\right)$ can be reconstructed with translational motion correction as follows:

$$
\mathrm{I}_{\mathrm{r}}=\mathrm{FK}(\mathrm{t}) \mathrm{e}^{\mathrm{i} 2 \pi \mathbf{k}(\mathbf{t}) \bullet \mathrm{A}(\mathbf{r}) \bullet \mathrm{S}(\mathrm{t})}
$$

where $(\mathrm{K})$ is the acquired $3 \mathrm{D}$ radial k-space data with coordinates $\mathbf{k}(\mathbf{t})=\left[\mathrm{k}_{\mathrm{x}}(\mathrm{t}), \mathrm{k}_{\mathrm{y}}(\mathrm{t}) \mathrm{k}_{\mathrm{z}}(\mathrm{t})\right]$, and $(\mathrm{F})$ is the nonuniform Fourier transform. It is important to note that by correcting $\mathrm{k}$-space with Eq. 1 the resulting image will be sharper in regions where $\mathbf{A}(\mathbf{r})$ reflects the underlying motion state (i.e. heart moving due to respiration) but become blurrier where it does not (i.e. static tissue). To address this, we reconstruct fNAV images (I) using localized linear translations [16]:

$$
\mathrm{I}=\sum_{\mathrm{r}} \mathrm{U}_{\mathrm{r}} \mathrm{I}_{\mathrm{r}}
$$

For each spatial location, a motion corrected image $I_{r}$ is reconstructed using Eq. 1 and the corresponding intensity value is added to the fNAV image using a mask $\left(U_{r}\right)$ which contains zeros everywhere except for the selected spatial location. In this way, every voxel in the resulting fNAV image is regionally corrected for respiratory motion. Similarly, we reconstruct fNAV displacement fields (D) as follows:

$$
\mathbf{D}=\sum_{\mathrm{r}} \mathrm{U}_{\mathrm{r}} \mathbf{A}(\mathbf{r})
$$

where each spatial location in D contains the corresponding fNAV coefficient.

To implement Eqs. 1-3, reconstruct fNAV images, and reconstruct fNAV displacement fields, we must estimate fNAV coefficients for every sampled spatial location. To do this in a computationally efficient manner, we propose a three-step approach. First, we estimate fNAV coefficients $\mathbf{A}(\tilde{\mathbf{r}})$ where $\tilde{\mathbf{r}}$ specifies a small region-of-interest containing the heart. Second, we use our estimate of $\mathbf{A}(\tilde{\mathbf{r}})$ to constrain solutions for the remaining fNAV coefficients. Third, we iteratively refine all of our fNAV coefficients by enforcing smooth transitions in the corresponding fNAV displacement fields.

\section{Estimating displacement of the heart}

Beginning with an initial estimate $\mathbf{A}(\tilde{\mathbf{r}})=[0,0,0]$, an intermediate image (I) is reconstructed using Eq. 1, and an image quality metric is used to iteratively improve the estimate for $\mathbf{A}(\tilde{\mathbf{r}})$ (Fig. 1c). This approach is well-known as autofocusing [23-26]. For the fNAV framework we use the previously validated metric of localized image gradient entropy $\mathrm{H}$ :

$$
\begin{aligned}
& \mathrm{H}=-\sum_{\mathrm{u}=\mathrm{x}-\frac{\mathrm{b}}{2}}^{\mathrm{x}+\frac{\mathrm{b}}{2}} \sum_{\mathrm{v}=\mathrm{y}-\frac{\mathrm{b}}{2}}^{\mathrm{y}+\frac{\mathrm{b}}{2}} \sum_{\mathrm{w}=\mathrm{z}-\frac{\mathrm{b}}{2}}^{\mathrm{z}+\frac{\mathrm{b}}{2}} \mathrm{p}_{\mathrm{uvw}} \log _{2}\left(\mathrm{p}_{\mathrm{uvw}}\right) \\
& \mathrm{p}_{\mathrm{uvw}}=\frac{\mathrm{guvw}_{\mathrm{uvw}} \mathrm{g}_{\mathrm{uvw}}}{\sum_{\mathrm{uvw}}=\sqrt{\left|\nabla_{\mathrm{u}} \tilde{\mathrm{I}}\right|^{2}+\left|\nabla_{\mathrm{v}} \tilde{\mathrm{I}}\right|^{2}+\left|\nabla_{\mathrm{w}} \tilde{\mathrm{I}}\right|^{2}}}
\end{aligned}
$$

where (b) defines the main lobe width of a separable low pass Hanning filter centered around a given spatial location, (p) is the normalized voxel intensity from the gradient $(\mathrm{g})$ of the intermediate image, and $\nabla$ is approximated by $1 \mathrm{D}$ finite differences $[16,17,19,26]$. The value of $\mathbf{A}(\widetilde{\mathbf{r}})$ that minimizes $\mathrm{H}$ is solved using a steepest descent algorithm where the gradient of $\mathrm{H}$ as a function of $\mathbf{A}(\tilde{\mathbf{r}})$ is approximated numerically:

\section{Estimating regional displacement}

The second step of the fNAV framework is to estimate fNAV coefficients for each voxel-wise spatial location. This step is necessarily to account for the non-linear motion of the heart and surrounding anatomy due to respiration wherein for example, the apex of the heart undergoes larger displacements than the base and great vessels. In principle we could perform the same iterative optimization described by the previous section and Fig. 1c, for every region of our 3D image. However, to reduce the computational burden, we instead form a bank $\left(B_{A}\right)$ of fNAV coefficients, image reconstructions $\left(B_{I}\right)$, and metric values $\left(B_{H}\right)$, corresponding to all of the tested values from the previous step, as well as a small grid of values near the optimum coefficients for a total of $(\mathrm{m})$ motion states (Fig. 1d). Now, in place of the steepest descent algorithm, the value of $\mathbf{A}(\mathbf{r})$ that minimizes $\mathrm{H}$ is efficiently solved by a sorting algorithm applied to $\mathrm{B}_{\mathrm{H}}$ and $\mathrm{B}_{\mathrm{A}}$. 


\section{Refining motion estimates}

The third and final step of the proposed framework is a joint optimization of the image metric and a smoothness constraint applied to the fNAV displacement fields (D) calculated from Eq. 4:

$$
\begin{aligned}
& \mathbf{A}(\mathbf{r})=\underset{\mathrm{m}}{\arg \min _{\mathrm{m}}}(\mathrm{H}+\lambda|\mathbf{C}(\mathbf{r})-\mathbf{A}(\mathbf{r})|) \\
& \mathbf{C}(\mathbf{r})=\frac{1}{\mathrm{c}^{3}} \sum_{\mathrm{u}=\mathrm{x}-\frac{\mathrm{c}}{2}}^{\mathrm{x}+\frac{\mathrm{c}}{2}} \sum_{\mathrm{v}=\mathrm{y}-\frac{\mathrm{c}}{2}}^{\mathrm{y}+\frac{\mathrm{c}}{2}} \sum_{\mathrm{w}=\mathrm{z}-\frac{\mathrm{c}}{2}}^{\mathrm{z}+\frac{\mathrm{c}}{2}} \mathbf{D}(\mathrm{uvw})
\end{aligned}
$$

where $\mathbf{C}(\mathbf{r})$ defines an average operator over a sliding window (c), applied to $\mathbf{D}$. The metric term $(\mathrm{H})$ is first solved using $B_{H}$ and $B_{A}$ as previously described, and $\mathbf{D}$ derived from that solution is iteratively updated using Eq. 4 (Fig. 1e). Finally, the refined estimates of $\mathbf{A}(\mathbf{r})$ are used to create a final 3D image with corrected respiratory motion (Fig. 1f).

\section{Tuning parameters}

The fNAV framework contains four user-defined "tuning" parameters: (i) the number of motion states $(\mathrm{m})$ added to $B_{A}, B_{I}$ and $B_{H}$, (ii) the width (b) of the localized image metric defined in Eq. 4, (iii) the width (c) of the smoothness constraint for the displacement fields defined in Eq. 6, and (iv) the weighting parameter $\lambda$ defined in Eq. 5 which provides a trade-off between the image metric and variation in the fNAV displacement fields. To investigate the impact of these parameters and determine their optimum values a comprehensive numerical simulation was developed and used for a non-exhaustive search focused on a trade-off between computation time and accuracy in the respiratory motion estimations.

\section{Numerical simulation framework}

Self-navigated ECG-triggered 3D radial data are synthesized using a numerical simulation developed for this work and inspired by the previously described MRXCAT approach [27]. In summary, high resolution $\left(1 \mathrm{~mm}^{3}\right) 3 \mathrm{D}$ volumes covering the chest are derived from the XCAT software which contains labels for each tissue of interest and produces realistic nonrigid cardiac and respiratory motion [28]. For a user-defined maximum level of respiratory motion, a total of 400 unique volumes are generated from XCAT and arranged into a five-dimensional (5D) array representing the 3D volume sampled across 20 phases of a full cardiac and respiratory cycle. The ground truth respiratory motion-fields are also generated from XCAT.

To create synthetic physiological data, cardiac cycles with realistic heart-rate variability and respiratory cycles with variability in both frequency and amplitude are generated, spanning the length of a synthetic CMR acquisition [29]. The user-defined acquisition parameters for the synthetic CMR sequence (Table 1) are chosen to match the in vivo data acquisitions described in the following section. For a given timepoint in the synthetic acquisition, a 3D volume representing the desired cardiac and respiratory phase is interpolated from the $5 \mathrm{D}$ array described above, the labelled tissues are converted to CMR contrast using relaxation properties from the literature and a bSSFP signal equation, and the inverse NUFFT, which contains simulated 3D coil sensitivities, is used to extract the desired radial readout [29]. Finally, complex gaussian noise is added to the synthetic $\mathrm{k}$-space data.

\section{Numerical simulation data acquisition}

To optimize the tuning parameters of the fNAV algorithm, validate displacement field estimations, and validate image reconstructions, 500 synthetic data sets were generated using the framework described above. The maximum respiratory motion amplitude ranged from 0-5 $\mathrm{mm}, 0-10 \mathrm{~mm}$, and $5-20 \mathrm{~mm}$ along the $x(L R), y$ (AP), and $\mathrm{z}(\mathrm{SI})$ directions, respectively.

\section{Healthy subject and patient data acquisition}

In this retrospective study, in vivo data were analyzed from 22 healthy subjects ( 7 female, $27 \pm 5$ years) and 549 cardiac patients (196 female, $58 \pm 18$ years) that were scanned at our institution between May 2014 and May 2016 on a $1.5 \mathrm{~T}$ clinical CMR scanner (MAGNETOM Aera, Siemens Healthineers, Erlangen, Germany) with the previously described prototype sequence and scan

Table 1 Simulated and in vivo acquisition parameters

\begin{tabular}{llll}
\hline Parameter & Simulations & Healthy subjects & Cardiac patients \\
\hline $\begin{array}{l}\text { Field-of-view } \\
\left(\mathrm{mm}^{3}\right)\end{array}$ & 220 & $200-210$ & $185-250$ \\
$\begin{array}{l}\text { Voxel size }\left(\mathrm{mm}^{3}\right) \\
\text { Excitation angle }\left(^{\circ}\right)\end{array}$ & 1 & $0.98-1.09$ & $0.88-1.15$ \\
$\begin{array}{l}\text { Repetition time } \\
\text { (ms) }\end{array}$ & 3.14 & $90-115$ & $60-115$ \\
$\begin{array}{l}\text { Echo time (ms) } \\
\text { Radial interleaves }\end{array}$ & 1.6 & $2.97-3.33$ & $2.5-5.27$ \\
$\begin{array}{l}\text { Profiles per inter- } \\
\text { leave }\end{array}$ & 26 & $1.57-1.76$ & $1.56-1.79$ \\
$\begin{array}{l}\text { Mean RR-interval } \\
\text { (ms) }\end{array}$ & $850-1500$ & $191-492$ & $199-2351$ \\
$\begin{array}{l}\text { Acquisition win- } \\
\text { dow (ms) }\end{array}$ & 82 & $25-69$ & $8-60$ \\
$\begin{array}{l}\text { Acquisition time } \\
\text { (s) }\end{array}$ & $475-843$ & $219-363$ & $583-1546$ \\
& & $50-71$ & $167-917$ \\
\end{tabular}


parameters listed in Table 1 [9]. Inclusion of patients was based on available raw data from the above time period without exclusion and therefore consisted of a range of pathologies including coronary artery disease and congenital heart disease. Patient heart rates were not controlled by pharmaceutical methods during the CMR examination. All participants provided written informed consent in accordance with our institutional guidelines.

\section{Image reconstruction}

To study the performance of $\mathrm{ANAV}$, all the simulated plus the 571 in vivo human data sets were reconstructed using 4 different approaches. In addition to the fNAV reconstruction described above and in Fig. 1, images were reconstructed without motion correction, using a previously reported method for $1 \mathrm{D}$ correction of self-navigation data [8], and using respiratory resolved XD-GRASP [14]. The XD-GRASP reconstruction matched previously reported parameters with total variation along the respiratory dimension (weight: 0.05 ), and solved using a conjugate gradient algorithm with 20 iterations [13, 14]. All reconstructions and analyses were performed in MATLAB (MathWorks, Inc., Natick, Massachusetts, USA) on a workstation equipped with two Intel Xeon CPUs, 512 GB of RAM, and an NVIDIA Tesla GPU. To increase the computation speed for fNAV reconstruction parallel processing was used to reconstruct individual volumes for the image bank. For all in vivo data sets, reconstruction times were recorded for RSN, XD-GRASP, and fNAV reconstructions and compared using paired $\mathrm{t}$-tests with the Bonferroni correction for multiple comparisons.

\section{Motion estimation analysis}

To assess the accuracy of fNAV estimates of 3D nonrigid motion, the optimized fNAV motion maps were visually compared to the ground truth values for the simulated data sets, and to motion maps derived from co-registering the end-inspiratory and end-expiratory bins of the XD-GRASP reconstructions for healthy subject and cardiac patient data using an open-source software for non-rigid image registration: NiftyReg [30]. The quality of the fNAV motion maps was then assessed by a linear fit and Pearson correlation coefficient for the $\mathrm{x}, \mathrm{y}$, and $\mathrm{z}$ components across four regions of interest approximately defined over the aortic arch, base of the heart, apex of the heart, and liver.

\section{Image quality analysis}

To assess the impact of motion correction on both simulated and in vivo image reconstruction, quantitative assessment of image quality across all reconstructions was assessed by the percentage vessel sharpness and visible vessel length of the left main + left anterior descending (LAD), left circumflex (LCX), and right (RCA) coronary arteries using Soap-Bubble [31]. For simulated images, this process was automated using the known vessel locations and therefore all 500 simulated data were assessed for vessel sharpness but not for vessel length. Conversely, all 22 healthy subjects were manually examined using Soap-Bubble as was a subset of 20 randomly selected cardiac patients. All measurements were statistically compared using paired $t$-tests with the Bonferroni correction for multiple comparisons.

For a given healthy subject or cardiac patient, images corresponding to the four reconstruction methods were placed in random order and the best reconstruction method was identified, based on the greatest overall visibility and sharpness of both the coronary arteries and ventricular septum, by a blinded expert reviewer (DP) with ten years experience in CCMRA. This analysis was performed on all 571 in vivo data sets and statistical significance was measured using a chi-square test. For the same subset of 20 cardiac patients chosen randomly for sharpness measurements, two blinded expert reviewers (DP and MS) graded the image quality by consensus for each reconstruction in a randomized order according to the following Likert scale: 0 -non-diagnostic, 1 -marked blurring, limited diagnostic value, 2 -moderate blurring, but diagnostic value, 3-mild blurring, good diagnostic value, 4 -excellent diagnostic value, with half grades assigned to images that fall between the 5 categories [32]. Statistical significance was measured using a paired $t$-test with the Bonferroni correction for multiple comparisons. Finally, two patients who underwent $\mathrm{x}$-ray coronary angiography were chosen to provide a qualitative comparison between $x$-ray angiography and fNAV images.

\section{Results}

\section{Tuning parameters}

Using the numerical simulation, the optimum size of the coefficient, image, and, metric banks corresponding to the number of motion states $(\mathrm{m})$ considered in the fNAV reconstruction, was determined by a linear distribution between the uncorrected state (i.e. $A_{x}=A_{y}=A_{z}=0$ ) and the optimum bulk fNAV coefficient as described in Fig. 1. This set-up was empirically observed to provide adequate representation of the nonrigid components of the ground truth simulated respiratory motion without the need for exhaustive searches. Additionally, the width of the localized image metric $(b=30 \mathrm{~mm})$, width of the smoothness constraint $(\mathrm{c}=7 \mathrm{~mm})$, and weighting parameter $(\lambda$ $=0.75)$ provided the highest accuracy for respiratory motion estimation. Additional file 1: Figure S1 provides a quantitative analysis of the tuning parameters as a function of root-mean-squared error calculated between 
fNAV reconstructions and ground truth for simulated data.

\section{Image reconstruction}

The reconstruction time for fNAV images (mean and standard deviation) was significantly higher than RSN $(6.1 \pm 2.1$ min vs $1.4 \pm 0.3$, min, $\mathrm{p}<0.025)$ but significantly lower than XD-GRASP $(6.1 \pm 2.1 \mathrm{~min}$ vs $25.6 \pm 7.1$, min, $\mathrm{p}<0.025)$. Variability in reconstruction time was primarily due to the number of active coil elements, matrix size, total number of acquired lines, and number of motion states present in the final fNAV reconstruction.

\section{Motion estimation analysis}

Figure 2 compares representative fNAV displacement fields to reference displacement fields during end-inspiration. For simulated data (Fig. $2 \mathrm{a}-\mathrm{c}$ ), the reference is the ground truth, whereas for healthy subject (Fig. $2 d-f$ ) and patient data (Fig. 2g-i) the reference corresponds to displacement fields derived from registering XD-GRASP images. Overall, there is good visual agreement between fNAV displacement fields and the corresponding reference displacement fields. An animated version of the fNAV and reference displacement fields demonstrating the full respiratory range is included as Additional file 2: Video S1.

For further evaluation of fNAV motion estimates, Fig. 3 plots the mean $x, y$, and $z$ components of the fNAV and reference displacement fields in ROIs containing the aortic arch, base and apex of the heart, and liver, for each simulated (Fig. 3a-d), healthy subject (Fig. 3e-h), and patient (Fig. 3i-l) data set. Overall, there is high correlation between the fNAV, and reference displacement estimates in particularly for the primary $\mathrm{z}$ direction in regions containing the heart. At the periphery of the image there is somewhat weaker correlation as well as for small values of displacement.

\section{Image quality analysis}

Figure 4 shows curved reformatted images of the LAD (blue arrows) and RCA (red arrows) from reconstructions of simulated data across three levels of respiratory motion. At low levels of motion (Fig. 4a: maximum amplitudes $\mathrm{LR}=0 \mathrm{~mm}, \mathrm{AP}=0 \mathrm{~mm}, \mathrm{SI}=10 \mathrm{~mm}$ ), the self-navigated (second column), XD-GRASP (third column), and fNAV (fourth column) provide clear visual improvement over the uncorrected images (first column) and the resulting images are similar to the ground truth reference (fifth column). However, for increasing levels of respiratory motion (Fig. 4b: maximum amplitudes $\mathrm{LR}=0 \mathrm{~mm}, \mathrm{AP}=5 \mathrm{~mm}, \mathrm{SI}=10 \mathrm{~mm}$, Fig. 4c: maximum amplitudes $\mathrm{LR}=5 \mathrm{~mm}, \mathrm{AP}=10 \mathrm{~mm}$, $\mathrm{SI}=20 \mathrm{~mm}$ ), only $\mathrm{fNAV}$ and XD-GRASP provide comparable visual image quality to the reference with less noise but more blur in the XD-GRASP reconstructions due to regularization.

Figure 5 provides a summary of vessel sharpness measurements across all 500 simulated data sets for the four reconstruction methods and three vessels. Overall, these simulated results corroborate previous studies showing that RSN provides a statistically significant increase in vessel sharpness relative to uncorrected images, that XD-GRASP images are significantly sharper than RSN, but we also see that fNAV leads to significantly sharper coronary arteries than all other reconstruction methods for the three vessels in keeping with the qualitative results shown in Fig. 4.

Figure 6 shows curved reformatted images of the RCA (Fig. 6a), LAD (Fig. 6b), and LCX (Fig. 6c) from three representative healthy subjects. Overall, these in vivo images corroborate the findings from the simulated data shown in Fig. 4 with fNAV providing the most consistent visualization of the full length of all three vessels. Similarly, Fig. 7 shows curved reformatted images of the RCA (Fig. 7a), and LAD + LCX (Fig. 7b, c), and LCX (Fig. 7c) from three representative patients. Once again, these images corroborate the findings from both the simulated data and healthy subjects with ANAV providing the best conspicuity of all three observed coronary arteries.

Figure 8 provides a summary of vessel sharpness and visible length measurements across all 22 healthy subjects and a subset of 20 cardiac patient data sets for the four reconstruction methods and three vessels. Overall, fNAV provides the sharpest vessel measurements with statistically significant increases relative to all other reconstructions in both the first $4 \mathrm{~cm}$ and full length of the RCA, LAD, and LCX $(\mathrm{p}<0.01)$. Conversely, vessel length measurements were relatively consistent across the four reconstruction methods.

In the assessment of which reconstruction method was selected as the best image by an expert reviewer across all 571 in vivo data sets, RSN $(n=51)$ was chosen more often than uncorrected $(n=15)$, XD-GRASP $(n=61)$ slightly more than RSN, but overall fNAV $(n=444)$ was observed to provide the best image quality in $78 \%$ of the data sets $(\mathrm{p}<0.001)$. Additionally, consensus grades of fNAV images $(2.6 \pm 0.6)$ were significantly higher $(\mathrm{p}<0.001)$ than uncorrected $(1.7 \pm 0.7), \mathrm{RSN}(1.9 \pm 0.6)$, and XD-GRASP $(1.8 \pm 0.8)$.

Finally, Fig. 9 demonstrates the feasibility of using fNAV to visualize coronary artery disease through qualitative comparison to $\mathrm{x}$-ray coronary angiography. In two patients, one with a significant stenosis (A-B) and one with a total occlusion (C-D), the disease is well visualized by the corresponding fNAV images. 

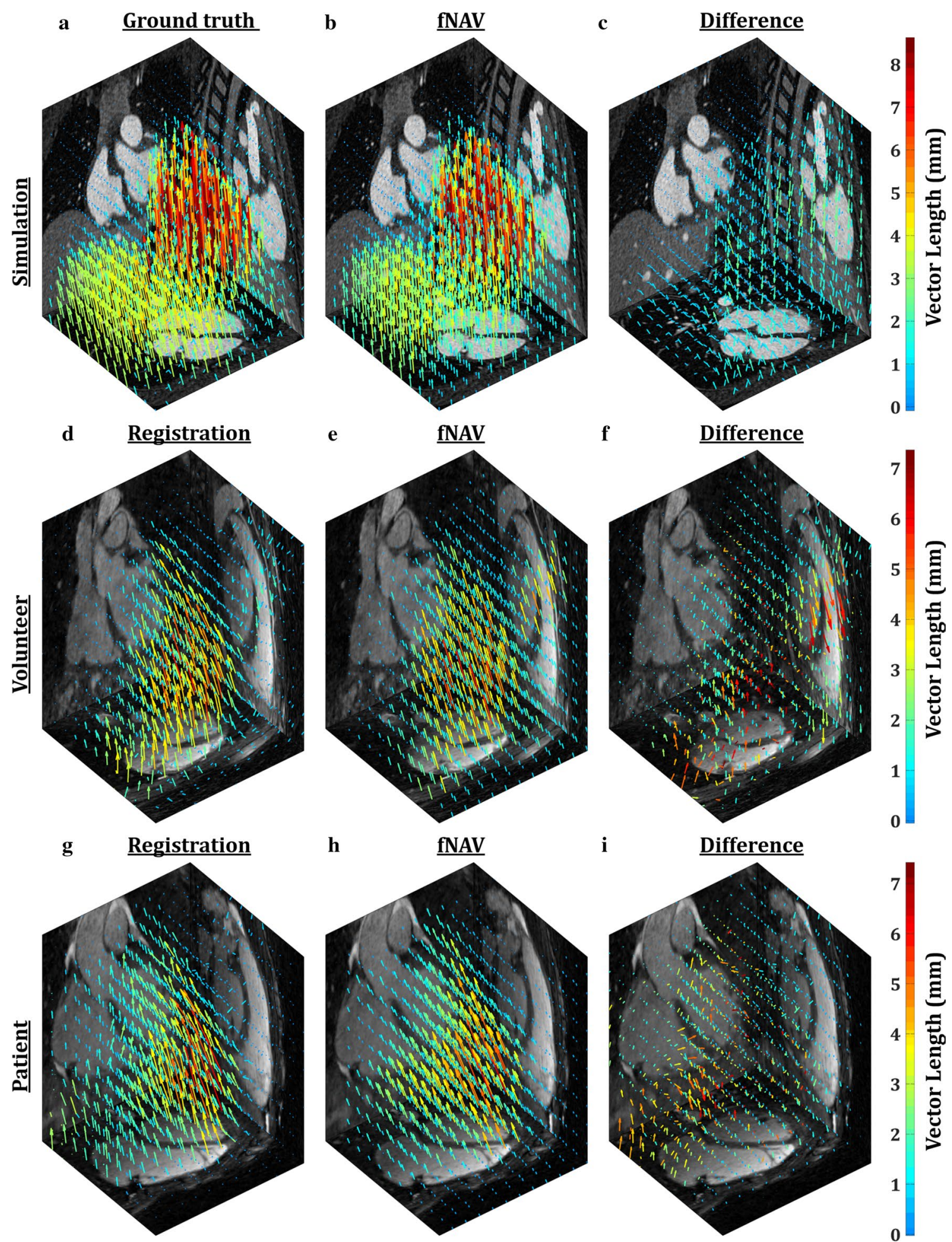

Fig. 2 Vector representation of respiratory motion. Displacement fields derived from ground truth (a) and focused navigation (fNAV) reconstructions of simulated data (b) demonstrate the nonrigid behavior of respiratory motion during end-inspiration, and excellent visual agreement is observed between fNAV and ground truth as shown by the difference image (c). Similarly, displacement fields derived from registering the frames of extradimensional golden-angle radial sparse parallel (XD-GRASP) reconstructions of a representative healthy subject (d) and cardiac patient $(\mathbf{g})$ are comparable to those derived using fNAV $(\mathbf{e}, \mathbf{h})$ as demonstrated by their corresponding difference images $(\mathbf{f}, \mathbf{i})$. An animated version of this figure is included as Additional file 2: Video S1 

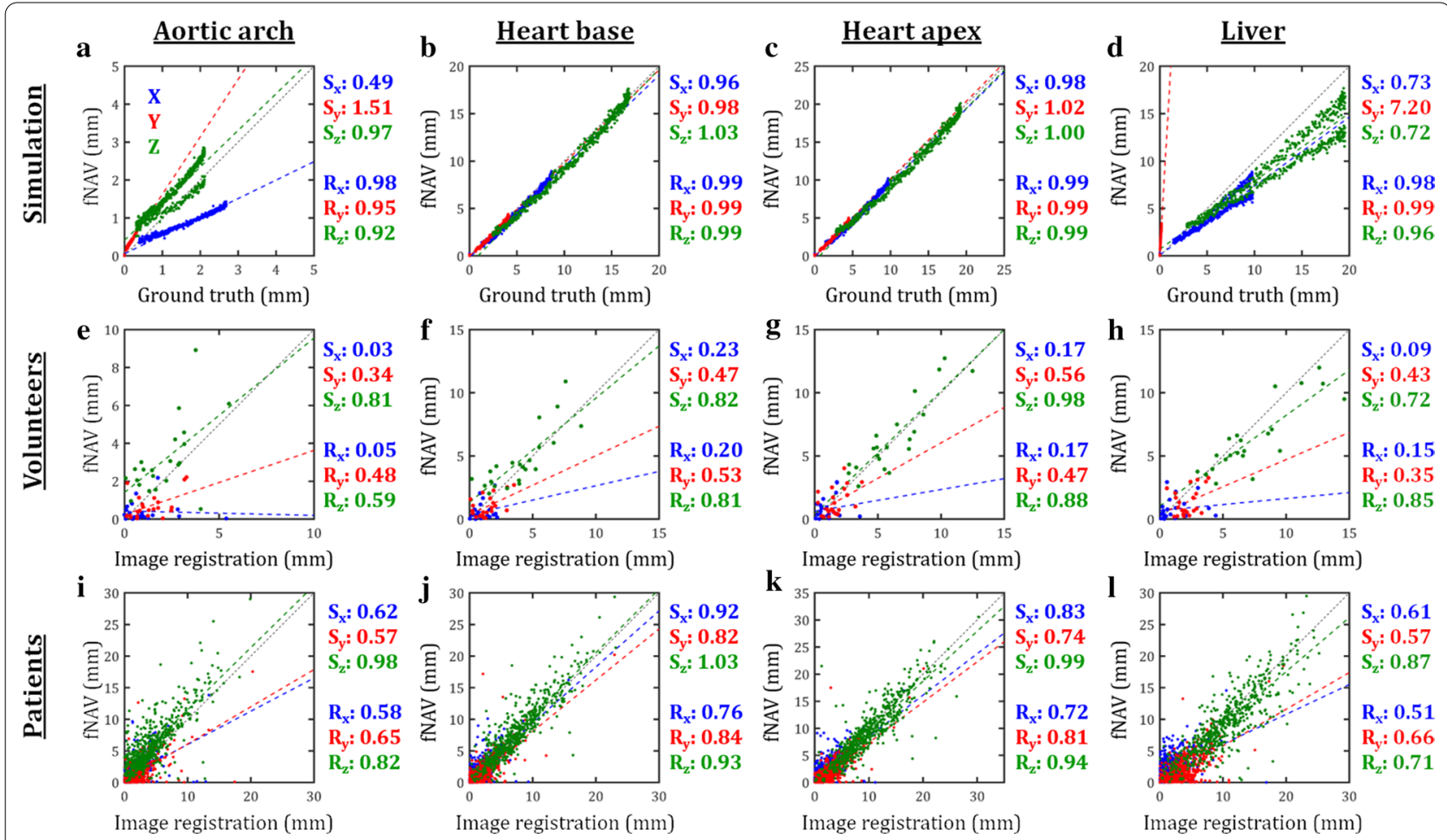

Image registration $(\mathrm{mm})$

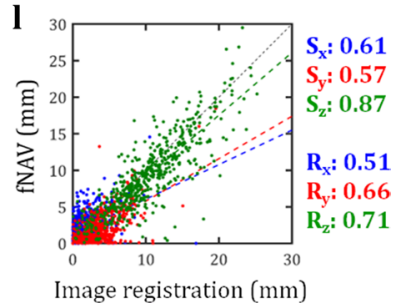

Fig. 3 Quantitative evaluation of respiratory motion estimation. The mean $\times$ (left-right; LR), y (anterior-posterior; AP), and z (superior-inferior; SI) components of respiratory motion estimated by fNAV measured in four regions of interest are shown for all 500 simulated data sets (a-d), all 22 healthy subject data sets $(\mathbf{e}-\mathbf{h})$ and all 549 cardiac patient data sets (i-I). Motion estimates derived from fNAV are compared to ground truth, and estimates derived from image registration for simulated and in vivo data, respectively. The results of a linear fit for each component is denoted by dash lines with the corresponding slopes and Pearson correlation coefficients given by $\left[S_{x^{\prime}} S_{y^{\prime}} S_{z}\right]$ and $\left[R_{x^{\prime}}, R_{y^{\prime}} R_{z}\right]$ respectively

\section{Discussion}

In this work, we developed and validated a novel framework for nonrigid, regional intra-acquisition correction of respiratory motion in self-navigated 3D whole-heart CCMRA scans. We showed that ANAV can accurately estimate and correct 3D respiratory motion yielding significant improvements in image quality and vessel sharpness relative to previously established approaches for $3 \mathrm{D}$ radial CCMRA. Validation of the fNAV framework and tuning parameters were performed in a comprehensive numerical simulation. These numerical results were then corroborated by in vivo reconstructions of 22 healthy subject and 549 cardiac patient data sets demonstrating the robustness of our proposed reconstruction framework.

Our results agree with previous studies that used the same self-navigated sequence to demonstrate improved image quality when comparing respiratoryresolved XD-GRASP reconstructions to motion corrected reconstructions using a $1 \mathrm{D}$ translational model. Yet in our study, motion correction using fNAV consistently yielded the highest values for objective vessel sharpness measurement when compared to both RSN and XD-GRASP in simulations, healthy subjects, and cardiac patients. Additionally, fNAV was selected as the best reconstruction with a statistically significant greater frequency in the more subjective yet blinded image quality assessment of the in vivo data and received statistically significantly higher grades in the Likert scale consensus scoring by two expert reviewers. This suggests that not only is a 3D model important for respiratory motion correction but that within the range of simulated and observed in vivo motion, XD-GRASP may suffer from blur due to uncorrected intra-bin motion or over-regularization. Additionally, fNAV reconstruction could be performed significantly faster than XD-GRASP $(6.1 \pm 2.1 \mathrm{~min}$ vs $25.6 \pm 7.1$, min, $\mathrm{p}<0.025)$. In principle, the motion information derived from fNAV could be used to inform intrabin correction of XD-GRASP reconstructions [33] or the fNAV displacement fields could be inserted directly into a compressed sensing or similar iterative denoising reconstruction [34]. Such approaches may allow for a reduction in scan time albeit at the likely cost of increased computation time during image reconstruction. 


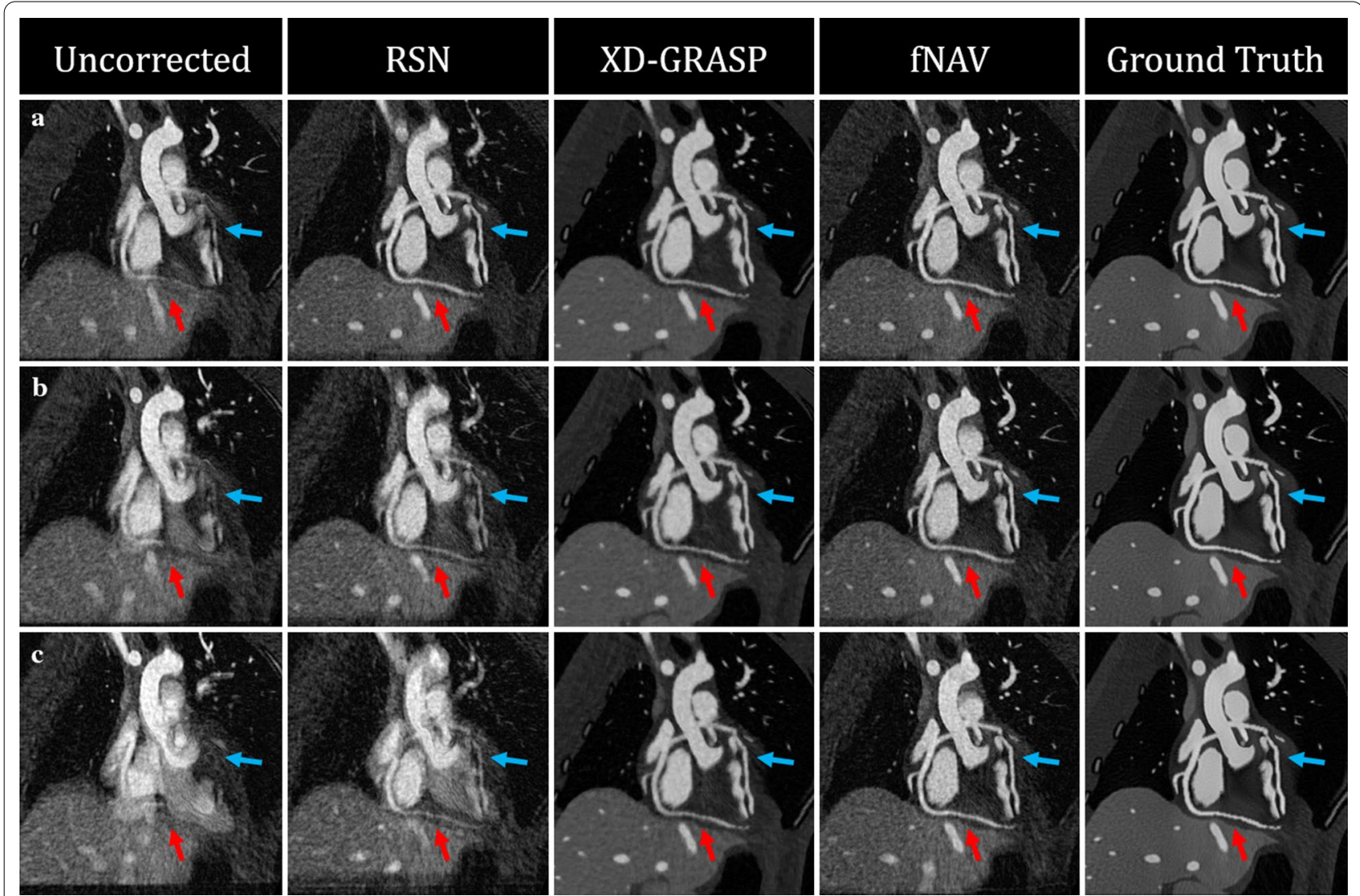

Fig. 4 Curved reformats of simulated data reconstructions at increasing levels of simulated motion. a Respiratory motion in only one dimension (maximum amplitudes $\mathrm{LR}=0 \mathrm{~mm}, \mathrm{AP}=0 \mathrm{~mm}, \mathrm{Sl}=10 \mathrm{~mm}$ ). $\mathbf{b}$ Respiratory motion in two dimensions (maximum amplitudes $\mathrm{LR}=0 \mathrm{~mm}$,

$\mathrm{AP}=5 \mathrm{~mm}, \mathrm{SI}=10 \mathrm{~mm}$ ). $\mathbf{c}$ Respiratory motion in the dimensions (maximum amplitudes $L R=5 \mathrm{~mm}, \mathrm{AP}=10 \mathrm{~mm}, \mathrm{SI}=20 \mathrm{~mm}$ ). Arrows denote the right (red) and left anterior descending (blue) coronary arteries
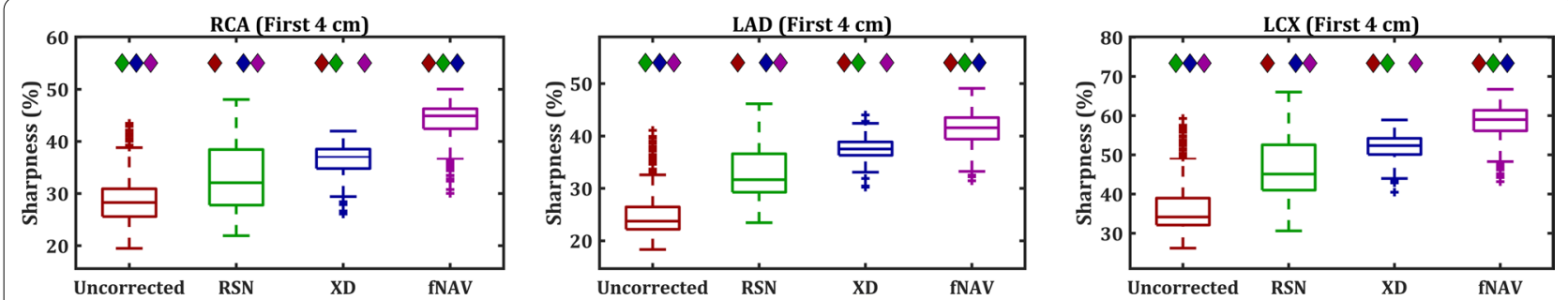

Fig. 5 Quantitative evaluation of vessel sharpness from simulated data. Percent vessel sharpness measured across the full length of the right coronary artery (RCA) (left), left anterior descending coronary artery (LAD) (middle), and left circumflex coronary artery (LCX) (right) in all 500 simulated data sets is shown. Colored diamonds denote statistically significant differences between the four reconstruction methods ( $p<0.01$ )

Overall, fNAV builds on previous work using autofocusing to correct motion in CMR images but this study is, to our knowledge, the first use of autofocusing for correcting free-breathing 3D radial self-navigated CCMRA data. Cheng et al. employed a localized image metric to approximate nonrigid motion correction as multiple translational corrections $[16,18]$. In their approach, socalled butterfly navigators were added to a 3D Cartesian acquisition providing motion estimates for each spatial direction and the signals from individual receiver coil channels were used to constrain the possible reconstructed motion states. In our work, the use of the selfnavigation signal allows us to estimate motion without significantly modifying the imaging sequence. Additionally, the method proposed in this work for joint optimization of the image metric and motion map smoothness is 


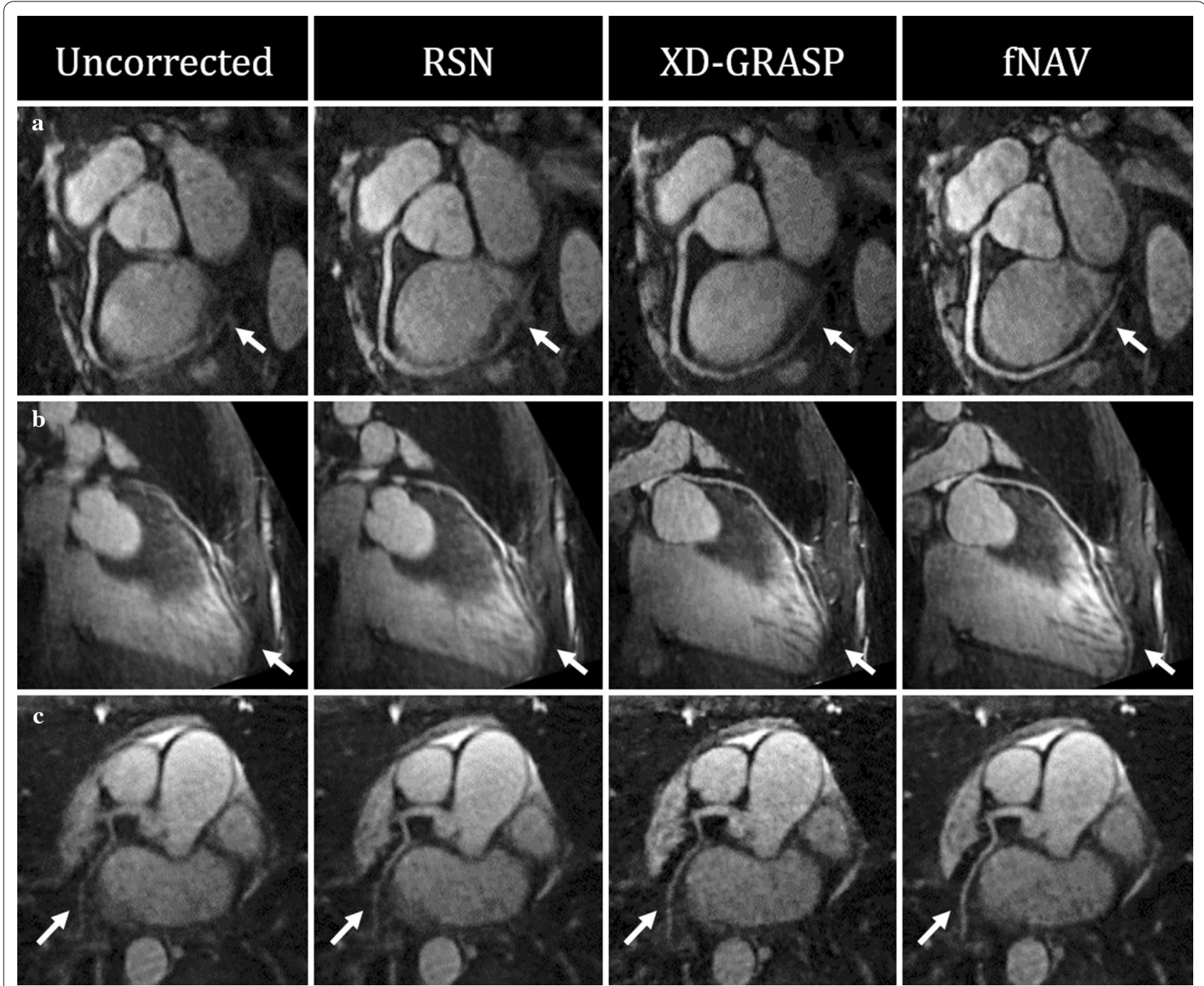

Fig. 6 Curved reformats of healthy subject data. The RCA (a), LAD (b), and LCX (c) are shown from a three representative volunteers with white arrows denoting regions of the vessels where image quality varies between the four reconstruction methods

similar to improvements discussed but not implement in previous work $[16,17]$.

A key feature of the proposed fNAV approach is the ability to convert a unitless $1 \mathrm{D}$ signal into regional, millimetric 3D displacements. We can do this by leveraging the rich $3 \mathrm{D}$ isotropic information in our resulting images which lend themselves to quantitative evaluation by our chosen image metric. Our results suggest that this works very well in regions containing cardiac anatomy where the visibility of complex structures is sensitive to respiratory motion and consequently can be captured by the image metric. Conversely, regions in the image with less complex structures or relatively uniform signal are less sensitive to change and may be misinterpreted by the image metric, which may weaken our ability to estimate
3D motion in regions such as the lungs or chest wall, for example.

Alternative methods for measuring motion during the acquisition include separately acquired two-dimensional (2D) [35-38], and three-dimensional (3D) [20, 39-44] image-based navigators. In particular, the works of Ingle et al. and Luo et al. have demonstrated that image navigators can be combined with autofocusing to reconstruct motion corrected data acquired using 3D spiral cones $[17,20,42]$. While, these multidimensional approaches inherently provide more information about the underlying motion, they require interruption of the imaging sequence to acquire the navigators and typically do not have the required resolution for direct estimation of nonrigid motion. For ECG-triggered coronary artery 


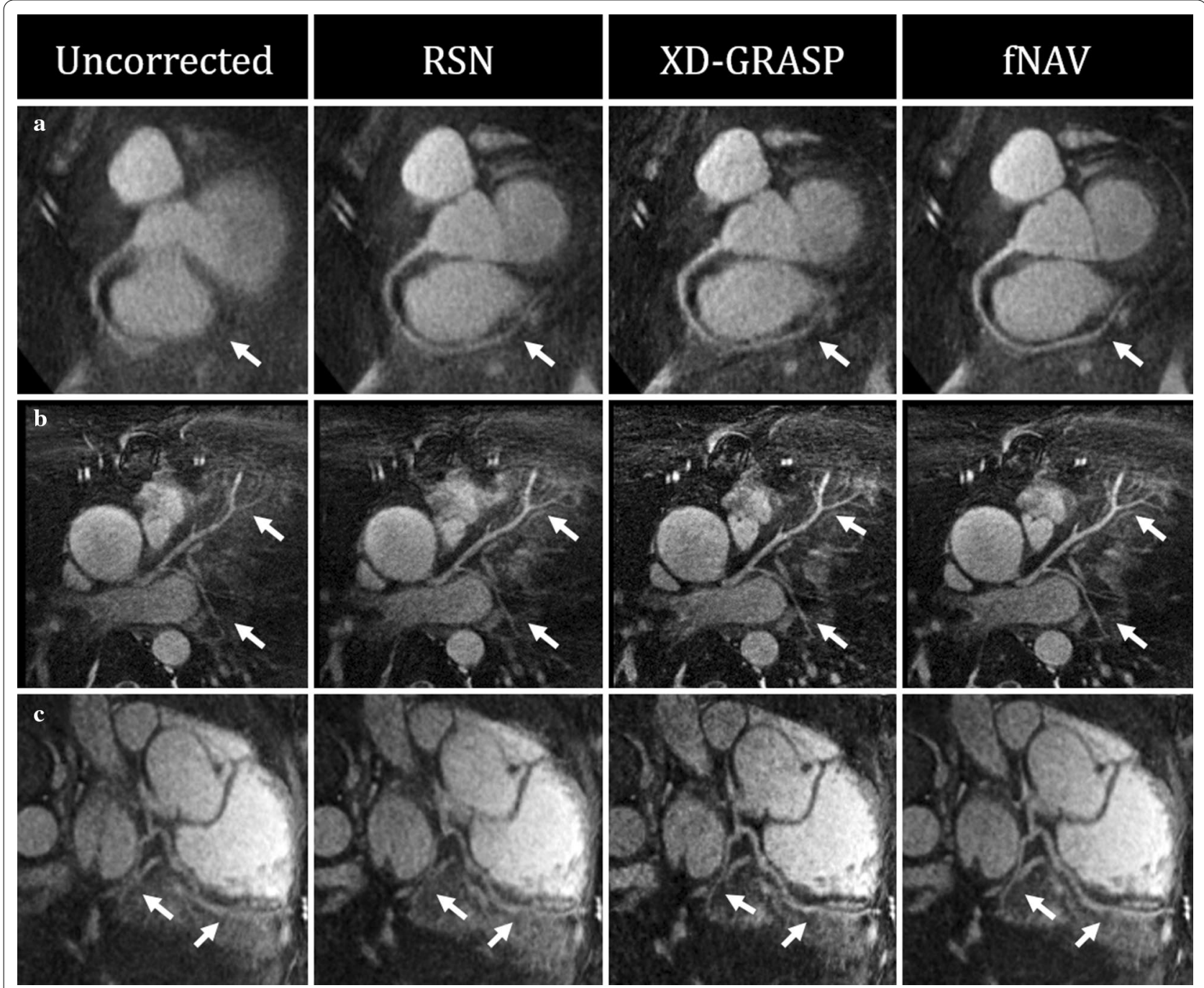

Fig. 7 Curved reformats of cardiac patient data. The RCA (a) and LAD $+L C X(\mathbf{b}, \mathbf{c})$ are shown from three representative patients with white arrows denoting regions of the vessels where image quality varies between the four reconstruction methods

imaging, the sequence is already interrupted and therefor a non-issue but recently proposed methods for "freerunning" CCMRA may benefit from the fNAV approach as they already periodically acquire SI readouts $[21,45$, 46]. The combination of fNAV and free-running may be applicable for total correction of respiratory motion thus producing cardiac motion-resolved $4 \mathrm{D}$ whole-heart CCMRA images. ANAV may also be used for intra-bin correction for cardiac- and respiratory motion-resolved 5D whole-heart images. 3D respiratory information can also be derived through nonrigid co-registration of respiratory resolved images [47-52] but may be limited to inter-acquisition corrections in image space unless combined with a generalized matrix model $[34,53,54]$ at the cost of increased computational complexity. For even further flexibility, our proposed approach could in principle be combined with a respiratory signal that is independent of the CMR sequence such as an external respiratory belt, or the recently proposed Pilot Tone Navigation System [55] which may in turn facilitate motion correction using other types of sequences.

Our results clearly demonstrate that fNAV provides improved image quality relative to previously described methods for 3D radial CCMRA and the two example comparisons to coronary angiography show the potential for evaluation of coronary disease using fNAV. Consequently, further studies are needed to investigate the impact of these improvements on clinically relevant parameters such as identification of stenoses and anomalous coronary arteries, as well as emerging techniques 

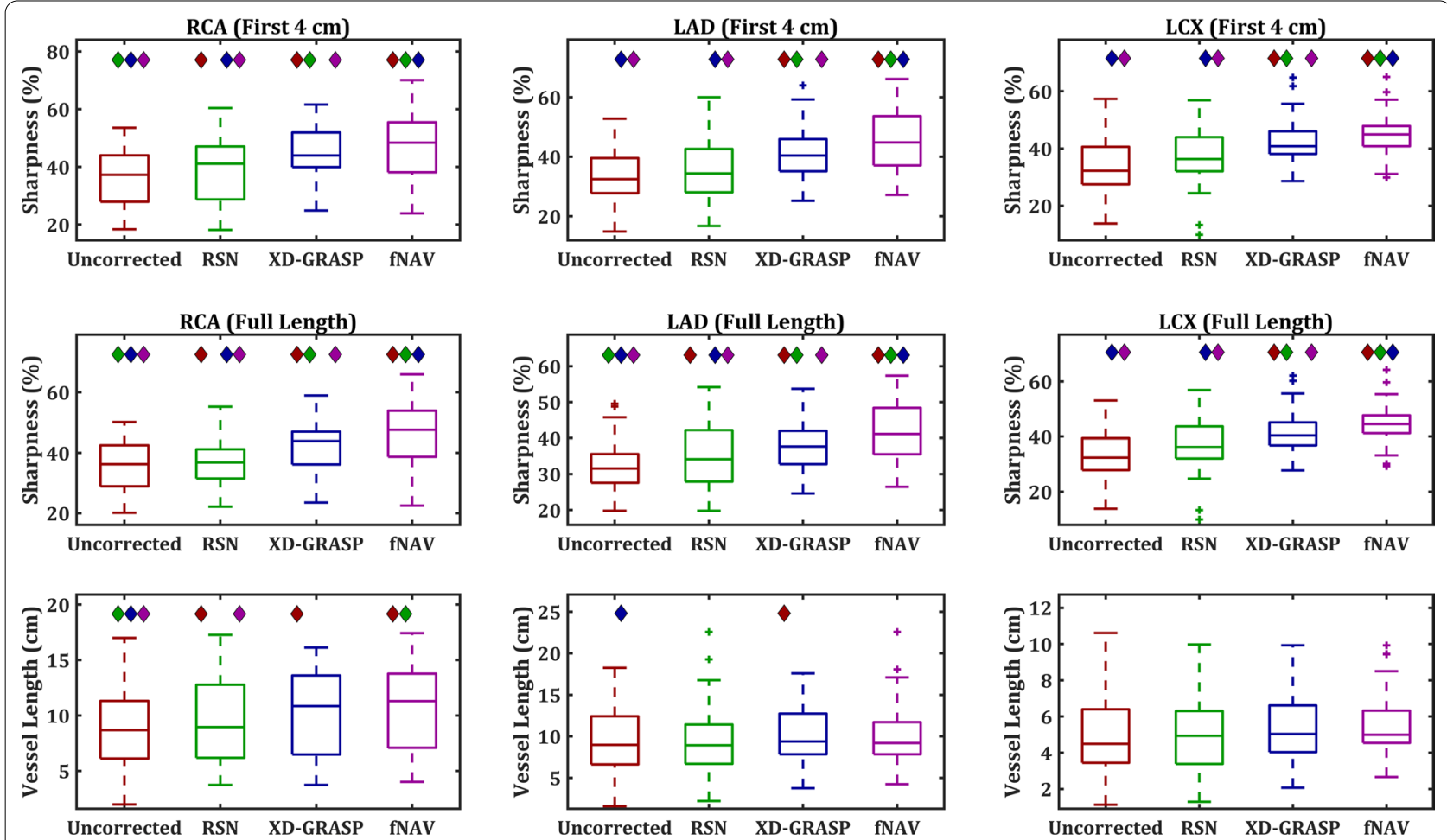

Fig. 8 Quantitative evaluation of vessel sharpness and length from all healthy subjects $(n=22)$ and a subset of cardiac patients ( $n=20)$. Percent vessel sharpness measured across the first $4 \mathrm{~cm}$ (top row) and full length (middle row) of the RCA (left), LAD (middle), and LCX (right) as well as vessel length (bottom row) is shown. Colored diamonds denote statistically significant differences between the four reconstruction methods

such as 3D modeling of the coronary anatomy for subsequent CCMRA-based fractional flow reserve estimations [56]. Furthermore, while the focus of this work was on coronary imaging, the employed 3D radial sequence provides whole-heart coverage with high isotropic spatial resolution and therefore the proposed fNAV approach may also be useful for evaluating other abnormalities in the cardiac anatomy such as congenital heart defects.

\section{Limitations and future directions}

Validation of the fNAV framework including the identification of optimum tuning parameters was largely performed using a numerical simulation developed for this work. The goal of the numerical simulation was to synthesize data with sequence parameters that match in vivo acquisitions and simulate a realistic range of respiratory motion and heart rate variability. Still, limitations of this simulation framework include the appearance of the coronary arteries which are relatively large and well defined when compared to in vivo CMR data sets because the XCAT models are derived from computed tomography data. Similarly, only one model of "normal" cardiac anatomy was used which does not reflect the variation we see in both healthy subjects and cardiac patients. Finally, the CMR physics simulated in this model consisted of a simplified equation for bSSFP contrast and complex coil sensitivities, neglecting the additional sources of artifact as well as effects of the T2-prep, saturation-slab, and fat saturation pulses that are used in the in vivo sequence.

Overall, the simulation provided a useful means of developing the fNAV framework, assessing the effects of motion and characterizing errors in the ANAV reconstructions, and the simulated results were generally corroborated by the large cohort of in vivo data that were analyzed. Nevertheless, improvements to the simulation framework or the addition of another ground truth measure may yield further improved tuning of the parameters for the fNAV framework. For example, an inherent tradeoff exists when selecting the width (b) of the localized image metric in Eq. 4. If the value of $b$ is too small, noiselike artifact can appear in the derived displacement fields and subsequent images while a large value of $b$ may fail to capture nonrigid deformation $[16,17,19]$. Additionally, the number of motion states considered for the final fNAV reconstruction has a large impact on the ability to assess non-linearities in the displacement fields. This is particularly evident in the displacement fields shown in Fig. 2a where motion of the chest wall, for example, is not well represented by the fNAV reconstructions of simulated data. 


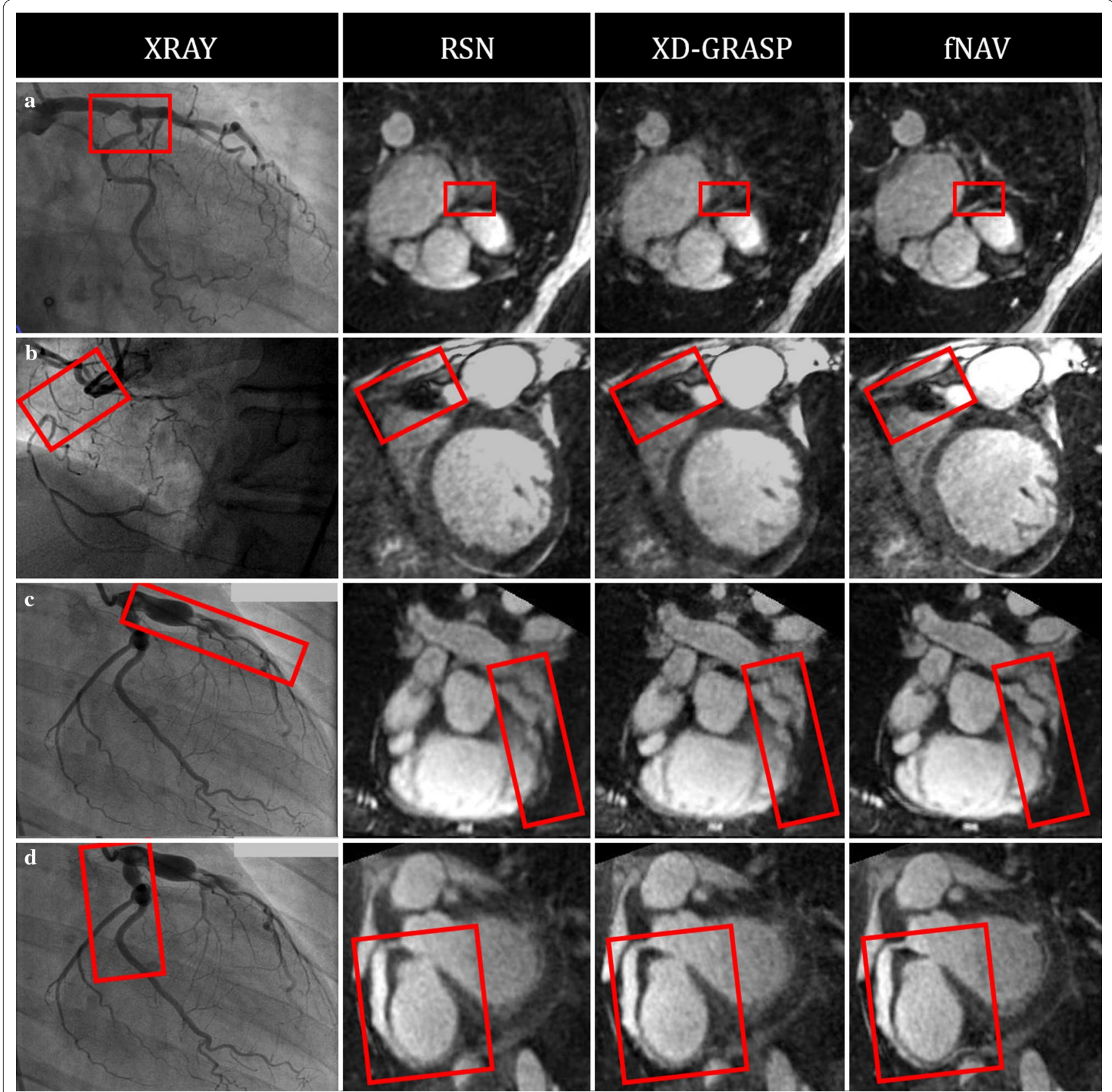

Fig. 9 Visualization of coronary artery disease using coronary angiography, RSN, XD-XD-GRASP, and fNAV. a Critical stenosis of the left main shown (b) chronic total occlusion of the proximal RCA. Aneurismal LAD (c) and RCA (d) in a patient with Kawasaki disease

Analysis of image quality across all four reconstruction methods was performed quantitatively using measurements of vessel sharpness and length and qualitatively via the best selected image for a given subject as identified by an expert reviewer. The rationale to choose only the best image was based on the need to evaluate 571 in vivo data sets. However, in subjects with poor overall scan quality or minimal motion it may be difficult to identify a single best image and therefore subjective image grading was also performed in a subset of 20 cardiac patients by two expert reviewers by consensus. Overall, both qualitative analyses agree with the overall narrative provided by the quantitative results that show fNAV provides the overall best image quality. 
In the current work, we focus on the estimation and correction of respiratory motion under the assumption that the cardiac motion is adequately compensated for using prospective triggering and an acquisition window chosen during the cardiac resting phase. However, heartrate variability, arrhythmias, and misidentification of the resting phase are not currently accounted for by fNAV and may degrade image quality. Still, for a given subject, the four reconstruction methods compared in this work were applied to the same data sets and therefore, residual cardiac motion is expected to have an equal effect on the resulting images. To overcome the limitations of prospective triggering, free-running approaches have been proposed for CMRA that acquire data throughout the cardiac cycle and, as mentioned above, may be compatible with the proposed fNAV framework $[21,45,46]$.

Finally, in the current model, we assume that different regions of the image are affected by different respiratory motion amplitudes, but we do not consider the phase. As such, hysteresis effects may contribute to errors in our estimated displacement fields and degrade our image quality. This may be addressed by including additional parameters in our fNAV model (i.e. respiratory phases coefficients for each spatial direction) but will potentially increase the computation time if more motion states need to be considered. Regardless, the inclusion of additional spatial information such as self-navigation signals from individual receiver coils may help constrain the potential motion states $[16,57]$.

\section{Conclusion}

Focused navigation is a promising technique for improving the quality of self-navigated free-breathing 3D radial whole-heart CCMRA. This novel approach to respiratory self-navigation can derive $3 \mathrm{D}$ nonrigid motion estimations from an acquired 1D signal yielding statistically significant improvement in image sharpness relative to 1D translational correction as well as XD-GRASP reconstructions. Using this approach motion corrected images can be reconstructed in 5-10 min compared to $\sim 20-30$ min using XD-GRASP, potentially facilitating integration in a clinical environment. Further study of the diagnostic impact of this technique is therefore warranted to evaluate its full clinical utility.

\footnotetext{
Abbreviations

1D: One-dimensional; 3D: Three-dimensional; 5D: Five-dimensional; AP: Anterior-posterior; bSSFP: Balanced steady state free precession; CCMRA: Coronary cardiovascular magnetic resonance angiography; CMR: Cardiovascular magnetic resonance; ECG: Electrocardiogram; fNAV: Focused navigation; LAD: Left anterior descending coronary artery; LCX: Left circumflex artery coronary artery; LR: Left-right; RCA: Right coronary artery; RSN: Radial self-navigated; SI: Superior-inferior; XD-GRASP: EXtra-Dimensional Golden-angle RAdial Sparse Parallel.
}

\section{Supplementary Information}

The online version contains supplementary material available at https://doi. org/10.1186/s12968-021-00717-4.

Additional file 1: Figure S1. Optimization of fNAV tunning parameters. Using our numerical simulation with the ground truth images as a reference, the root-mean-square error was calculated for fNAV reconstructions as a function of A) the width of the localized image metric, B) the width of the smoothness constraint, $C$ ) the weighting parameter $\lambda$. The parameter values $(b=30 \mathrm{~mm}, c=7 \mathrm{~mm}, \lambda=0.75)$ that minimized the error was used for all subsequent reconstructions.

Additional file 2: Video S1. Displacement fields derived from ground truth (A) and fNAV reconstructions of simulated data (B) and the absolute difference $(C)$ overlayed on the ground truth respiratory resolved images. Similarly, displacement fields derived from registering the frames of XD-GRASP reconstructions $(D, G)$ fNAV $(E, H)$ and the absolute difference $(F, I)$ from a healthy volunteer and patient respectively overlayed on the respiratory resolved XD-GRASP reconstructions.

\section{Acknowledgements}

Not applicable

\section{Authors' contributions}

$\mathrm{CWR}, \mathrm{JH}, \mathrm{DP}$, and MS contributed to the study design and analysis. CWR and GR developed the FNAV reconstruction algorithm. JH and DP acquired the healthy volunteer data. AGP and JS acquired the patient data and contributed to patient-specific analysis. All authors contributed to and approved the final manuscript.

\section{Funding}

Schweizerischer Nationalfonds zur Förderung der Wissenschaftlichen Forschung, Grant/Award Number: \#173129.

\section{Availability of data and materials}

The datasets and algorithms used and analyzed during the current study are available from the corresponding author on reasonable request.

\section{Ethics approval and consent to participate}

All subjects provided informed written consent, including permission to publish anonymized data, as part of a study approved by the CHUV institutional review board.

\section{Competing interests}

The PhD studies of JH are supported financially by Siemens Healthcare (Erlangen, Germany). DP is an employee of Siemens Healthcare AG (Lausanne, Switzerland). MS receives non-monetary research support from Siemens Healthcare (Erlangen, Germany). JS receives research support by Bayer Healthcare, Switzerland.

\section{Author details}

${ }^{1}$ Department of Radiology, Lausanne University Hospital (CHUV) and University of Lausanne (UNIL), Rue de Bugnon 46, BH-7-84, 1011 Lausanne, Switzerland. ${ }^{2}$ Advanced Clinical Imaging Technology (ACIT), Siemens Healthcare AG, Lausanne, Switzerland. ${ }^{3}$ Division of Cardiology, Lausanne University Hospital (CHUV), Lausanne, Switzerland. ${ }^{4}$ Director CMR-Center, Lausanne University Hospital (CHUV), Lausanne, Switzerland. ${ }^{5}$ Faculty of Biology and Medicine, University of Lausanne (UNIL), Lausanne, Switzerland. ${ }^{6}$ Center for Biomedical Imaging (CIBM), Lausanne, Switzerland.

Received: 26 September 2020 Accepted: 28 January 2021

Published online: 29 March 2021

References

1. Hauser TH, Manning WJ. The promise of whole-heart coronary MRI. Curr Cardiol Rep. 2008;10:46-50. https://doi.org/10.1007/s11886-008-0010-8. 
2. Stuber M, Weiss RG. Coronary magnetic resonance angiography. J Magn Reson Imaging. 2007;26:219-34. https://doi.org/10.1002/jmri.20949.

3. Lanzer P, Botvinick EH, Schiller NB, et al. Cardiac imaging using gated magnetic resonance. Radiology. 1984;150:121-7. https://doi.org/10.1148/ radiology.150.1.6227934.

4. Ehman RL, Felmlee JP. Adaptive technique for high-definition MR imaging of moving structures. Radiology. 1989;173:255-63. https://doi. org/10.1148/radiology.173.1.2781017.

5. Wang Y, Rossman PJ, Grimm RC, Riederer SJ, Ehman RL. Navigator-echobased real-time respiratory gating and triggering for reduction of respiration effects in three-dimensional coronary MR angiography. Radiology. 1996;198:55-60. https://doi.org/10.1148/radiology.198.1.8539406.

6. Stehning C, Bornert P, Nehrke K, Eggers H, Stuber M. Free-breathing whole-heart coronary MRA with 3D radial SSFP and self-navigated image reconstruction. Magn Reson Med. 2005;54:476-80. https://doi. org/10.1002/mrm.20557.

7. Piccini D, Littmann A, Nielles-Vallespin S, Zenge MO. Spiral phyllotaxis: the natural way to construct a 3D radial trajectory in MRI. Magn Reson Med. 2011;66:1049-56. https://doi.org/10.1002/mrm.22898.

8. Piccini D, Littmann A, Nielles-Vallespin S, Zenge MO. Respiratory selfnavigation for whole-heart bright-blood coronary MRI: Methods for robust isolation and automatic segmentation of the blood pool. Magn Reson Med. 2012;68:571-9. https://doi.org/10.1002/mrm.23247.

9. Piccini D, Monney P, Sierro C, et al. Respiratory self-navigated postcontrast whole-heart coronary MR angiography: initial experience in patients. Radiology. 2014;270:378-86. https://doi.org/10.1148/radiol.13132045.

10. Monney P, Piccini D, Rutz T, et al. Single centre experience of the application of self navigated $3 \mathrm{D}$ whole heart cardiovascular magnetic resonance for the assessment of cardiac anatomy in congenital heart disease. $J$ Cardiovasc Magn Reson. 2015;17:55. https://doi.org/10.1186/s1296 8-015-0156-7.

11. Albrecht MH, Varga-Szemes A, Schoepf UJ, et al. Coronary artery assessment using self-navigated free-breathing radial whole-heart magnetic resonance angiography in patients with congenital heart disease. Eur Radiol. 2018;28:1267-75. https://doi.org/10.1007/s00330-017-5035-1.

12. Heerfordt J, Stuber M, Maillot A, Bianchi V, Piccini D. A quantitative comparison between a navigated Cartesian and a self-navigated radial protocol from clinical studies for free-breathing 3D whole-heart bSSFP coronary MRA. Magn Reson Med. 2020;84:157-69. https://doi. org/10.1002/mrm.28101.

13. Feng L, Axel L, Chandarana H, Block KT, Sodickson DK, Otazo R. XDGRASP: golden-angle radial MRI with reconstruction of extra motion-state dimensions using compressed sensing. Magn Reson Med. 2016;75:77588. https://doi.org/10.1002/mrm.25665.

14. Piccini D, Feng L, Bonanno G, et al. Four-dimensional respiratory motionresolved whole heart coronary MR angiography. Magn Reson Med. 2016. https://doi.org/10.1002/mrm.26221.

15. Stroud RE, Piccini D, Schoepf UJ, et al. Correcting versus resolving respiratory motion in free-breathing whole-heart MRA: a comparison in patients with thoracic aortic disease. Eur Radiol Exp. 2019;3:29. https:// doi.org/10.1186/s41747-019-0107-4.

16. Cheng JY, Alley MT, Cunningham CH, Vasanawala SS, Pauly JM, Lustig M. Nonrigid motion correction in 3D using autofocusing with localized linear translations. Magn Reson Med. 2012;68:1785-97. https://doi. org/10.1002/mrm.24189.

17. Ingle RR, Wu HH, Addy NO, et al. Nonrigid autofocus motion correction for coronary MR angiography with a 3D cones trajectory. Magn Reson Med. 2014;72:347-61. https://doi.org/10.1002/mrm.24924.

18. Cheng JY, Zhang T, Ruangwattanapaisarn N, et al. Free-breathing pediatric MRI with nonrigid motion correction and acceleration. J Magn Reson Imaging. 2015;42:407-20. https://doi.org/10.1002/jmri.24785.

19. Cheng JY, Hanneman K, Zhang T, et al. Comprehensive motion-compensated highly accelerated 4D flow MRI with ferumoxytol enhancement for pediatric congenital heart disease. J Magn Reson Imaging. 2016:43:135568. https://doi.org/10.1002/jmri.25106.

20. Luo J, Addy NO, Ingle RR, et al. Nonrigid motion correction with 3D image-based navigators for coronary MR angiography. Magn Reson Med. 2016;00:1-10. https://doi.org/10.1002/mrm.26273.

21. Di Sopra LPD, Coppo S, Stuber MYJ. An automated approach to fully self-gated free-running cardiac and respiratory motion-resolved $5 D$ whole-heart MRI. Magn Reson Med. 2019;00(1):15.
22. Ginami G, Bonanno G, Schwitter J, Stuber M, Piccini D. An iterative approach to respiratory self-navigated whole-heart coronary MRA significantly improves image quality in a preliminary patient study. Magn Reson Med. 2016;75:1594-604. https://doi.org/10.1002/mrm.25761.

23. Atkinson D, Hill DLG, Stoyle PNR, Summers PE, Keevil SF. Automatic correction of motion artifacts in magnetic resonance images using an entropy focus criterion. IEEE Trans Med Imaging. 1997;16:903-10. https:// doi.org/10.1109/42.650886.

24. Atkinson D, Hill DLG, Stoyle PNR, et al. Automatic compensation of motion artifacts in MRI. Magn Reson Med. 1999;41:163-70. https:// doi.org/10.1002/(SICI)1522-2594(199901)41:1\%3c163:.AID-MRM23 \%3e3.0.CO;2-9.

25. Manduca A, Mcgee KP, Welch EB, Felmlee JP, Ehman RL. Autofocusing of clinical shoulder MR images for correction of motion artifacts. Methods. 1998;25:598-605

26. McGee KP, Manduca A, Felmlee JP, Riederer SJ, Ehman RL. Image metricbased correction (Autocorrection) of motion effects: analysis of image metrics. J Magn Reson Imaging. 2000;11:174-81.

27. Wissmann L, Santelli C, Segars WP, Kozerke S. MRXCAT: Realistic numerical phantoms for cardiovascular magnetic resonance. J Cardiovasc Magn Reson. 2014;16:63. https://doi.org/10.1186/s12968-014-0063-3.

28. Segars WP, Sturgeon G, Mendonca S, Grimes J, Tsui BMW. 4D XCAT phantom for multimodality imaging research. Med Phys. 2010;37:4902. https:// doi.org/10.1118/1.3480985.

29. Roy CW, Marini D, Segars WP, Seed M, Macgowan CK. Fetal XCMR: a numerical phantom for fetal cardiovascular magnetic resonance imaging. J Cardiovasc Magn Reson. 2019;21:29. https://doi.org/10.1186/s1296 8-019-0539-2.

30. Modat M, Ridgway GR, Taylor ZA, et al. Fast free-form deformation using graphics processing units. Comput Methods Programs Biomed. 2010:98:278-84. https://doi.org/10.1016/j.cmpb.2009.09.002.

31. Etienne A, Botnar RM, van Muiswinkel AMC, Boesiger P, Manning WJ, Stuber M. ?Soap-Bubble? visualization and quantitative analysis of 3D coronary magnetic resonance angiograms. Magn Reson Med. 2002;48:658-66. https://doi.org/10.1002/mrm.10253.

32. Piccini D, Demesmaeker R, Heerfordt J, et al. Deep learning to automate reference-free image quality assessment of whole-heart MR images. Radiol Artif Intell. 2020;2:e190123. https://doi.org/10.1148/ryai.20201 90123.

33. Correia T, Ginami G, Cruz G, et al. Optimized respiratory-resolved motioncompensated 3D Cartesian coronary MR angiography. Magn Reson Med. 2018;80:2618-29. https://doi.org/10.1002/mrm.27208.

34. Odille F, Vuissoz P-A, Marie P-Y, Felblinger J. Generalized reconstruction by inversion of coupled systems (GRICS) applied to free-breathing MRI. Magn Reson Med. 2008;60:146-57. https://doi.org/10.1002/mrm.21623.

35. Dewan $\mathrm{M}$, Hager GD, Lorenz CH. Image-based coronary tracking and beat-to-beat motion compensation: feasibility for improving coronary MR angiography. Magn Reson Med. 2008;60:604-15. https://doi.org/10.1002/ mrm.21663.

36. Henningsson M, Koken P, Stehning C, Razavi R, Prieto C, Botnar RM. Whole-heart coronary MR angiography with $2 \mathrm{D}$ self-navigated image reconstruction. Magn Reson Med. 2012;67:437-45. https://doi. org/10.1002/mrm.23027.

37. Henningsson M, Shome J, Bratis K, Vieira MS, Nagel E, Botnar RM. Diagnostic performance of image navigated coronary CMR angiography in patients with coronary artery disease. J Cardiovasc Magn Reson. 2017;19:68. https://doi.org/10.1186/s12968-017-0381-3.

38. Kawaji K, Spincemaille P, Nguyen TD, et al. Direct coronary motion extraction from a 2D fat image navigator for prospectively gated coronary MR angiography. Magn Reson Med. 2014;71:599-607. https://doi. org/10.1002/mrm.24698.

39. Scott AD, Keegan J, Firmin DN. Beat-to-beat respiratory motion correction with near 100\% efficiency: a quantitative assessment using high-resolution coronary artery imaging. Magn Reson Imaging. 2011;29:568-78. https://doi.org/10.1016/j.mri.2010.11.004.

40. Moghari MH, Roujol S, Henningsson M, et al. Three-dimensional heart locator for whole-heart coronary magnetic resonance angiography. Magn Reson Med. 2014;71:2118-26. https://doi.org/10.1002/mrm.24881.

41. Powell J, Prieto C, Henningsson M, Koken P, Botnar R. CMRA with $100 \%$ navigator efficiency with 3D self navigation and interleaved 
scanning. J Cardiovasc Magn Reson. 2014;16:08. https://doi. org/10.1186/1532-429X-16-S1-O8.

42. Addy NO, Ingle RR, Luo J, et al. 3D image-based navigators for coronary MR angiography. Magn Reson Med. 2017;77:1874-83. https://doi. org/10.1002/mrm.26269.

43. Keegan J, Gatehouse PD, Yang G-Z, Firmin DN. Non-model-based correction of respiratory motion using beat-to-beat 3D spiral fat-selective imaging. J Magn Reson Imaging. 2007;26:624-9. https://doi.org/10.1002/ jmri.20941.

44. Wu HH, Gurney PT, Hu BS, Nishimura DG, McConnell MV. Free-breathing multiphase whole-heart coronary MR angiography using image-based navigators and three-dimensional cones imaging. Magn Reson Med. 2013;69:1083-93. https://doi.org/10.1002/mrm.24346.

45. Bastiaansen JAM, Piccini D, Sopra L, et al. Natively fat-suppressed 5D whole-heart MRI with a radial free-running fast-interrupted steady-state (FISS) sequence at 1.5 T and 3 T. Magn Reson Med. 2019. https://doi. org/10.1002/mrm.27942.

46. Masala N, Bastiaansen JAM, Sopra L, et al. Free-running 5D coronary MR angiography at $1.5 \mathrm{~T}$ using LIBRE water excitation pulses. Magn Reson Med. 2020;8:221. https://doi.org/10.1002/mrm.28221.

47. Buerger C, Schaeffter T, King AP. Hierarchical adaptive local affine registration for fast and robust respiratory motion estimation. Med Image Anal. 2011;15:551-64. https://doi.org/10.1016/j.media.2011.02.009.

48. Schmidt JFM, Buehrer M, Boesiger P, Kozerke S. Nonrigid retrospective respiratory motion correction in whole-heart coronary MRA. Magn Reson Med. 2011;66:1541-9. https://doi.org/10.1002/mrm.22939.

49. Pang J, Bhat $\mathrm{H}$, Sharif B, et al. Whole-heart coronary MRA with $100 \%$ respiratory gating efficiency: self-navigated three-dimensional retrospective image-based motion correction (TRIM). Magn Reson Med. 2014;71:67-74. https://doi.org/10.1002/mrm.24628.

50. Aitken AP, Henningsson M, Botnar RM, Schaeffter T, Prieto C. 100\% Efficient three-dimensional coronary MR angiography with two-dimensional beat-to-beat translational and bin-to-bin affine motion correction. Magn Reson Med. 2015;74:756-64. https://doi.org/10.1002/mrm.25460.
51. Pang J, Chen Y, Fan Z, et al. High efficiency coronary MR angiography with nonrigid cardiac motion correction. Magn Reson Med. 2016;76:1345-53. https://doi.org/10.1002/mrm.26332.

52. Cruz G, Atkinson D, Henningsson M, Botnar RM, Prieto C. Highly efficient nonrigid motion-corrected 3D whole-heart coronary vessel wall imaging. Magn Reson Med. 2016;1:1. https://doi.org/10.1002/mrm.26274.

53. Batchelor PG, Atkinson D, Irarrazaval P, Hill DLG, Hajnal J, Larkman D. Matrix description of general motion correction applied to multishot images. Magn Reson Med. 2005;54:1273-80. https://doi.org/10.1002/ mrm.20656.

54. Odille F, Cîndea N, Mandry D, Pasquier C, Vuissoz P-A, Felblinger J. Generalized MRI reconstruction including elastic physiological motion and coil sensitivity encoding. Magn Reson Med. 2008;59:1401-11. https://doi. org/10.1002/mrm.21520.

55. Speier P, Fenchel M, Rehner R. PT-Nav: a novel respiratory navigation method for continuous acquisitions based on modulation of a pilot tone in the MR-receiver. Magn Reson Mater Phys Biol Med. 2015;28:1-135. https://doi.org/10.1007/s10334-015-0487-2.

56. Hair J, Oshinski J. MRI-derived virtual fractional flow reserve (vFFR): preliminary results. In: Proceedings 27th scientific meeting, international society for magnetic resonance in medicine. 2019. p. 2143.

57. Odille F, Uribe S, Batchelor PG, Prieto C, Schaeffter T, Atkinson D. Modelbased reconstruction for cardiac cine MRI without ECG or breath holding. Magn Reson Med. 2010;63:1247-57. https://doi.org/10.1002/mrm.22312.

\section{Publisher's Note}

Springer Nature remains neutral with regard to jurisdictional claims in published maps and institutional affiliations.
Ready to submit your research? Choose BMC and benefit from:

- fast, convenient online submission

- thorough peer review by experienced researchers in your field

- rapid publication on acceptance

- support for research data, including large and complex data types

- gold Open Access which fosters wider collaboration and increased citations

- maximum visibility for your research: over $100 \mathrm{M}$ website views per year

At BMC, research is always in progress.

Learn more biomedcentral.com/submissions 